\title{
Juristinnen machen Karriere - wir stellen sie vor
}

\author{
Kristiane Weber-Hassemer, Frankfurt am Main
}

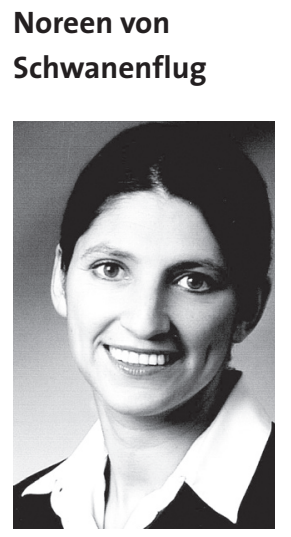

Noreen von

Schwanenflug

Vorsitzende des

LV Hessen

\begin{abstract}
Was hat Sie bewegt Jura zu studieren? Was war Ihr Berufswunsch?

Ursprünglich wollte ich nur politische Wissenschaften und Soziologie studieren. Ich habe dann aber eingesehen, dass die Rechtswissenschaften eine solidere Basis bildeten, politische Wissenschaften und Soziologie studierte ich aber als Nebenfächer. Ich war in dieser Zeit eine der wenigen Frauen, die Jura studierten. Auch viele Hochschullehrer waren damals der Ansicht, Jura sei kein Studium für Frauen, weil ihnen das logische Denken fehle. Ich bekam im 4. Semester meinen Sohn und musste unter Beweis stellen, dass ein Studium auch mit Kind möglich war. Ursprünglich wollte ich - wie seinerzeit wohl viele Träumer - im diplomatischen Dienst tätig sein. Ich war allerdings auch für andere Bereiche offen. Meine praktische Arbeit begann ich dann als Rechtsanwältin.
\end{abstract}

Sie haben eine vielseitige Karriere durchlaufen: Rechtsanwältin, Richterin, Vors. Richterin am OLG, Pressesprecherin des OLG Frankfurt, Staatssekretärin im Hessischen Ministerium der Justiz und für Europaangelegenheiten, Mitglied des NER und seit 2005 Vorsitzende des NER. Haben Sie Ihre Karriere geplant? Haben Sie gezögert, den Vorsitz des NER zu übernehmen?

Meine Karriere habe ich zunächst überhaupt nicht geplant. Erst nachdem ich in Frankfurt Richterin wurde, wollte ich es innerhalb der Justiz zu etwas bringen. Nein, ich habe nicht gezögert, den Vorsitz des NER zu übernehmen. Ich habe mich gefreut, dass ich mich neuen Herausforderungen stellen kann.

Wer hat Sie in Ihrer beruflichen Laufbahn unterstützt? Frauen und Männer gleichermaßen? Haben Sie Frauennetzwerke genutzt?

In erster Linie haben mich Männer in meiner Karriere unterstützt. Dies liegt wohl daran, dass zu Beginn meiner beruflichen Laufbahn Frauen noch nicht so in den oberen Etagen vertreten waren, die mir hätten helfen können voranzukommen. Es gab männliche Gerichtspräsidenten, männliche Minister und Staatssekretäre.

Frauennetzwerke waren damals noch nicht in dem Umfang vorhanden, dass man sie praktisch nutzen konnte. Meine juristische Karriere habe ich also ohne Frauennetzwerke durchlaufen. Später wurde ich sicher auch gefördert von Netzwerken, z. B. für die Position im Ethikrat. Ich selbst habe dann aber Frauen gezielt gefördert, als ich die Möglichkeiten dazu hatte.

Wie kamen Sie zu dem Bereich Ethik? Was interessiert Sie daran besonders?

Während meiner Mitgliedschaft in der Ethikkommission der Landesärztekammer von 1990 bis 1995 kam ich zu dem Bereich Ethik. Die Schnittstelle zwischen Ethik, Recht und Naturwissenschaft fand und finde ich sehr spannend. In der Ethikkommission ging es seinerzeit um die klinische Medikamentenforschung am Menschen.

Wie sieht Ihr Arbeitsalltag aus? Sie arbeiten in interdisziplinär besetzten Gremien. Wie hoch ist dort der Frauenanteil in den Führungspositionen? Als Vorsitzende Richterin eines Haftsenats war ich im Regelfall täglich am Gericht. Dies ließ sich gerade noch mit der Mitgliedschaft im NER vereinbaren.

Die Arbeit als Vorsitzende des NER setzt aber sehr viel mehr Präsenz in Berlin voraus. Ich muss viel reisen und Kontakte zu anderen Ethikräten innerhalb und außerhalb Europas pflegen. Außerdem muss ich natürlich Vorträge an Hochschulen, Schulen, Wissenschaftsorganisationen und Bürgerforen halten. Ich arbeite aber auch aktiv in den einzelnen Arbeitsgruppen für die Stellungnahmen

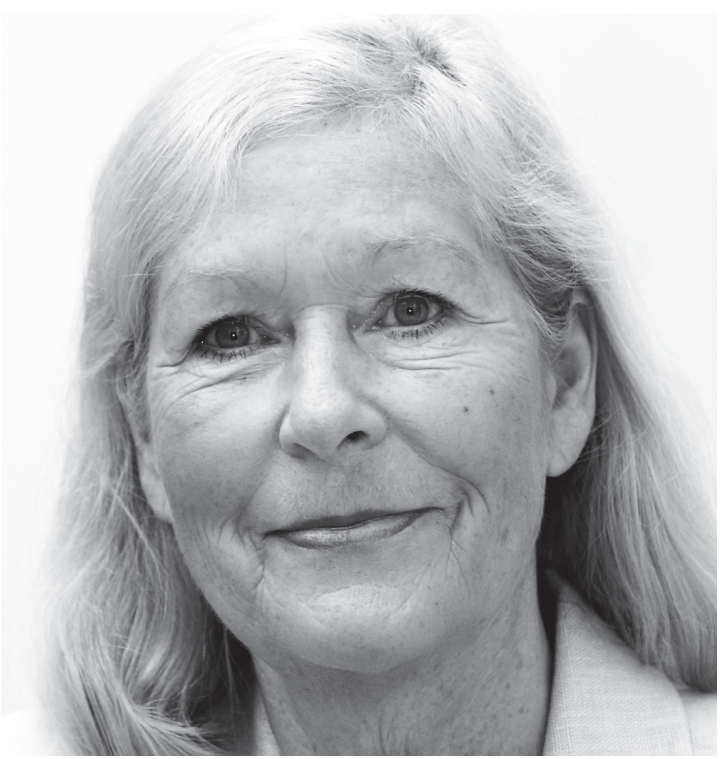


des NER mit. Einmal im Monat finden - im Regelfall öffentliche - Plenarsitzungen statt. Dort werden in den Sitzungen auch die Stellungnahmen erörtert. Wir veranstalten daneben auch Tagungen und abendliche Diskussionsforen zu speziellen bioethischen Fragestellungen und geben einen Infobrief heraus. Das alles erfordert viel Planung und Vorbereitung, dabei werde ich aber sehr von der Geschäftsstelle unterstützt.

Es gab im ersten vierjährigen Turnus eine stellvertretende Vorsitzende. Gegenwärtig sind meine beiden Stellvertreter männlich. Von den 24 Mitgliedern sind acht weiblich. In der Schweiz existiert eine Quotenregelung für die Berufung der Mitglieder. Der NER wurde durch einen Kabinettsbeschluss errichtet, eine Quote war nicht vorgesehen. Aber auch das neue Ethikratgesetz vom 16. Juli 2007, das zum 1. August 2007 in Kraft trat und die Rechtsgrundlage des künftigen Deutschen Ethikrates bildet, verzichtet auf eine Quotenregelung. In der Geschäftsstelle des NER sind etwas mehr Frauen als Referentinnen und Sachbearbeiterinnen beschäftigt, das Geschlechterverhältnis ist inzwischen aber nahezu ausgeglichen.

Berücksichtigen Sie auch Genderaspekte bei Ihrer täglichen Arbeit? Vermitteln Sie eine weibliche Sicht?

Die medizinischen und ethischen Auswirkungen müssen häufig geschlechtsspezifisch differenziert werden. Die Frauenperspektive ist meiner Ansicht nach sehr wichtig, gerade wenn zwischen der Selbstbestimmung und ihrem etwaigen Missbrauch abgewogen wird. Im NER wird allerdings innerhalb der Mitgliedschaft darüber kontrovers diskutiert. Es zeichnet sich keine „weibliche Position“ $\mathrm{ab}$.

\section{Was wird für Frauen in der Geschäftsstelle des NER getan?}

Wir bemühen uns um möglichst familien- und frauenfreundliche Arbeitsbedingungen. Mitarbeiterinnen und Mitarbeiter können - sofern sie dennoch voll erreichbar sind - für wissenschaftliche Recherchen teilweise zuhause arbeiten oder, falls erforderlich, auch ihr Kind mal in die Geschäftsstelle mitbringen. Ich halte es für wichtig, dass wir in den Rahmenbedingungen flexibel sind. Die Solidarität unter den Mitarbeiterinnen und Mitarbeitern ist beeindruckend, dies erleichtert es.

\section{Glauben Sie, dass heute gezielte Frauenförderung noch notwendig ist oder ist sie ein alter Zopf?}

Frauenförderung ist in gewissen Bereichen notwendig und klug und Netzwerke sind sehr wichtig. Heute werden die Frauen durch die Netzwerke endlich sichtbar. Frauen sollen ihre Begabung wahrnehmen können, dabei kann eine Hilfestellung auch sehr praktisch aussehen, wie z. B. die Bezahlung von Haushaltshilfen oder Tagesmüttern aus Fonds, die der oder die Vorgesetzte organisiert hat, wie ich es im Bekanntenkreis erlebt habe.
Kristiane Weber-Hassemer, geb. 1939 in Berlin, studierte von 1960 bis 1964 Rechtswissenschaften, politische Wissenschaften und Soziologie an den Universitäten Tübingen und Hamburg. Das erste Staatsexamen absolvierte sie 1964 in Hamburg. Nach einer Babypause absolvierte sie das zweite Staatsexamen 1969 in Hamburg. Von 1969 bis 1976 arbeitete sie als Rechtsanwältin in Hamburg mit den Schwerpunkten Arbeitsrecht, Gesellschaftsrecht und Strafrecht. 1976 wurde sie Richterin in Frankfurt am Main in der ordentlichen Gerichtsbarkeit. Von 1986 an arbeitete sie als Richterin am Oberlandesgericht Frankfurt am Main. Von 1990 bis 2002 war sie Mitglied im Frankfurter Arbeitskreis für Strafrecht. Sie war von 1990 bis
1995 Mitglied der Ethikkommission der Landesärztekammer. Sie gehört seit 1998 dem Studienkreis für Presserecht und Pressefreiheit an. 1994 wurde Kristiane Weber-Hassemer Vorsitzende Richterin am Oberlandesgericht Frankfurt am Main und Pressesprecherin des OLG. Von 1995 bis 1999 arbeitete sie als Staatssekretärin im Hessischen Ministerium der Justiz und für Europaangelegenheiten. Nach dem Regierungswechsel in Hessen war sie von 1999 bis 2004 als Vorsitzende Richterin eines Strafsenates am OLG Frankfurt tätig. 1999 war sie Vorsitzende des Arbeitsstabes Justizreform des djb.

2001 erfolgte ihre Berufung zum Mitglied des Nationalen Ethikrates (NER) durch Beschluss des
Bundeskabinetts. 2005 erfolgte die Wiederberufung von Kristiane Weber-Hassemer zum Mitglied des NER und die Wahl zur Vorsitzenden des NER. Das Interview führte Noreen von Schwanenflug, Vorsitzende des LV Hessen im Oktober 2007. 
Was war Ihr größtes Erfolgserlebnis? Was war Ihre größte Niederlage?

Mein größtes Erfolgserlebnis war, dass ich trotz aller Widerstände im weiten Familienkreis ein gutes 1. Staatsexamen machte, entgegen aller Vorurteile.

Es gab natürlich einige Niederlagen in meiner beruflichen Laufbahn. Eine gewisse Enttäuschung war für mich, dass ich wegen der Wahl in Hessen meine Tätigkeit als Staatssekretärin im Hessischen Ministerium der Justiz und für Europaangelegenheiten nicht fortführen konnte, aber eine Niederlage war das natürlich nicht.

\section{Was raten Sie Juristinnen, die Beruf und Familie kombinieren möchten? \\ Ich rate Juristinnen, die es zu etwas bringen möchten, nicht zu lange zu pausieren. Die Kinderpause sollte nicht wesent- lich länger als ein Jahr sein.}

\section{Was raten Sie Frauen, die in Führungspositionen aufsteigen wollen? Gibt es eine Strategie dafür?}

Ich denke, Frauen, die eine Führungsposition anstreben, sollten bereit sein, jedenfalls für einige Zeit auch persönliche Opfer zu bringen. D.h. das Privatleben wird sich eine Zeit lang dem Berufsleben unterordnen müssen. Frauen, die Karriere machen möchten, sollten sich sehr genau die konkreten Bedingungen für die einzelne Position anschauen.
Netzwerke können sehr hilfreich sein, um in Führungspositionen zu gelangen, aber auch Fürsprecher bzw. Mentoren innerhalb einer bestimmten Organisation sind wertvolle Helfer für das Fortkommen.

Was verbindet Sie mit dem djb? Sie waren Vorsitzende des Arbeitsstabs Justizreform. Konnten Sie die Arbeit im djb auch beruflich nutzen?

Ich bin erst 1997, also sehr spät in den djb eingetreten, ohne dass ich hierfür einen triftigen Grund nennen könnte. Mich beeindruckt die Professionalität der Frauen im djb und die Solidarität untereinander. Ich finde sehr gut, dass alle Arbeitsbereiche im djb gepflegt werden. Meine Arbeit im djb als Vorsitzende des Arbeitsstabes Justizreform konnte ich beruflich indirekt nutzen, insofern ich mich gründlich in die Materie eingearbeitet habe. Das half, mir über die Justizstrukturen Klarheit zu verschaffen, was ich für meine Arbeit dringend brauchte. Sie machte mir viel Freude.

\section{Zum Abschluss: Was macht Ihnen privat Freude?}

Ich reise gern und bin fasziniert von Landschaften und Orten. Garten- oder Hausgestaltung bzw. Hausumbauten machen mir Spaß. Ich interessiere mich sehr für Architektur und Kunst, und wenn meine Zeit es mir erlaubt, gehe ich unterwegs in Museen. Außerdem haben wir zwei Katzen!!

Frau Weber-Hassemer, vielen Dank für das Gespräch!

\section{Impressum}

\author{
Schriftleitung: \\ Anke Gimbal, Rechtsassessorin (V.i.S.d.P.) \\ Juliane Lindner
}

Redaktionsanschrift:

Deutscher Juristinnenbund e. V.

Anklamer Str. 38

10115 Berlin

Telefon: 030/ 443270-0

Telefax: 030/ 443270-22

E-Mail: geschaeftsstelle@djb.de

\section{Druck und Verlag:}

Nomos Verlagsgesellschaft mbH \& Co. KG

Waldseestr. 3-5

D-76530 Baden-Baden

Telefon: 07221/ 2104-O

Telefax: 07221/2104-27

\author{
Anzeigenverwaltung und \\ Anzeigenannahme: \\ Sales friendly, Verlagsdienstleistungen \\ Bettina Roos \\ Siegburgerstr. 123 \\ D-53229 Bonn \\ Telefon: 0228/ 97898-0 \\ Telefax: 0228/ 97898-20 \\ E-Mail: roos@sales-friendly.de
}

Die Zeitschrift sowie alle in ihr enthaltenen einzelnen Beiträge und Abbildungen sind urheberrechtlich geschützt. Jede Verwertung, die nicht ausdrücklich vom Urheberrechtsgesetz zugelassen ist, bedarf der vorherigen Zustimmung des Verlages.

Namentlich gekennzeichnete Artikel müssen nicht die Meinung des Herausgebers oder der Schriftleitung wiedergeben. Unverlangt eingesendete Manuskripte - für die keine Haftung übernommen wird - gelten als Veröffentli- chungsvorschlag zu den Bedingungen des Verlags. Es werden nur unveröffentlichte Originalarbeiten angenommen. Die Verfasser erklären sich mit einer nicht sinnentstellenden redaktionellen Bearbeitung einverstanden.

Erscheinungsweise:

vierteljährlich

Bezugspreis 2008:

jährlich 48,-€, Einzelheft 13,-€

Alle Preise zzgl. Vertriebs-Direktbeordnungsgebühren inkl. MWSt.;

Bestellungen nehmen entgegen:

Der Buchhandel und der Verlag; Kündigung jeweils drei Monate zum Kalenderjahresende. Zahlungen jeweils im Voraus an: Nomos Verlagsgesellschaft, Postbank Karlsruhe, Konto 7363651 (BLZ 66010075) oder Stadtsparkasse Baden-Baden, Konto 5002266 (BLZ 66250030). 